\title{
IRAS 01005+7910, A High Galactic Latitude Post-AGB Star
}

Jingyao $\mathrm{Hu}$

National Astronomical Observatories, Chinese Academy of Sciences 20A Datun Road, Beijing 100012, China

hjy@bao.ac.cn

$\mathrm{Hu}$ et al (1993) have selected a sample of proto-planetary nebula candidates based on the IRAS color-color diagram. IRAS $01005+7910$ is one object of this sample. We have observed this object photometrically (Table 1) and spectroscopically (Fig. 1). From its spectral type of B2I and optical color of $B-$ $V=0.23$, we can derive the reddening as $E(B-V)=0.39$ and interstellar/circumstellar absorption $A_{v}=1.20$. If it is a normal B-type supergiant, the distance module will be $m-M=16.05$ and distance $d=16.2 \mathrm{kpc}$. Due to the galactic latitude $b=16.6$, it should be located at about 4.6 kpcabove the galactic plane. This does not fit with normal B-type supergiant and we considered that it is a post-AGB star located at high galactic latitude. Recently Hrivnak et al (2000) found that this object shows carbon-rich features in the infrared. A paper on observations, reduction and discussions of this object has been submitted to the Chinese Journal of Astronomy and Astrophysics.

\section{Table 1. BVRI magnitude and IRAS flux of IRAS 01005+7910}

\begin{tabular}{ll}
\hline \hline magnitude & IRAS flux \\
\hline $\mathrm{B}=11.08$ & 3.90Jy (12 micron) \\
$\mathrm{V}=10.85$ & $24.23 \mathrm{Jy}$ (25 micron) \\
$\mathrm{R}=10.68$ & 10.07Jy (60 micron) \\
$\mathrm{I}=10.50$ & $2.42 \mathrm{Jy}(100$ micron $)$ \\
\hline
\end{tabular}

\section{References}

Hrivnak B.J., Volk K., Kwok, S. 2000, ApJ 535, 275

Hu J.Y., Slijkhuis S., de Jong T., Jiang B.W., 1993 A\&AS 100, 413 


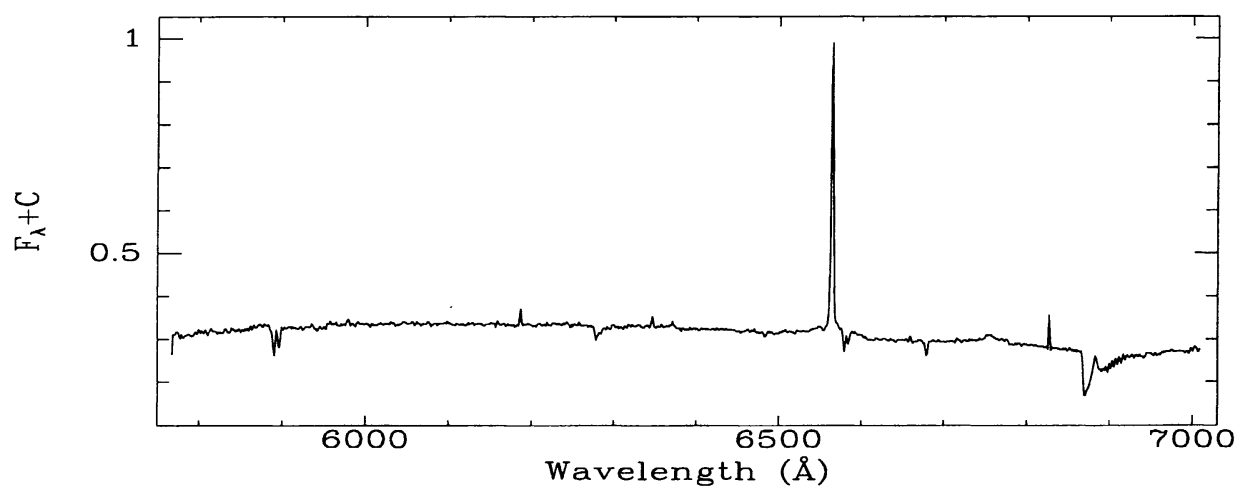

Figure 1. Optical spectrum of IRAS 01005+7910

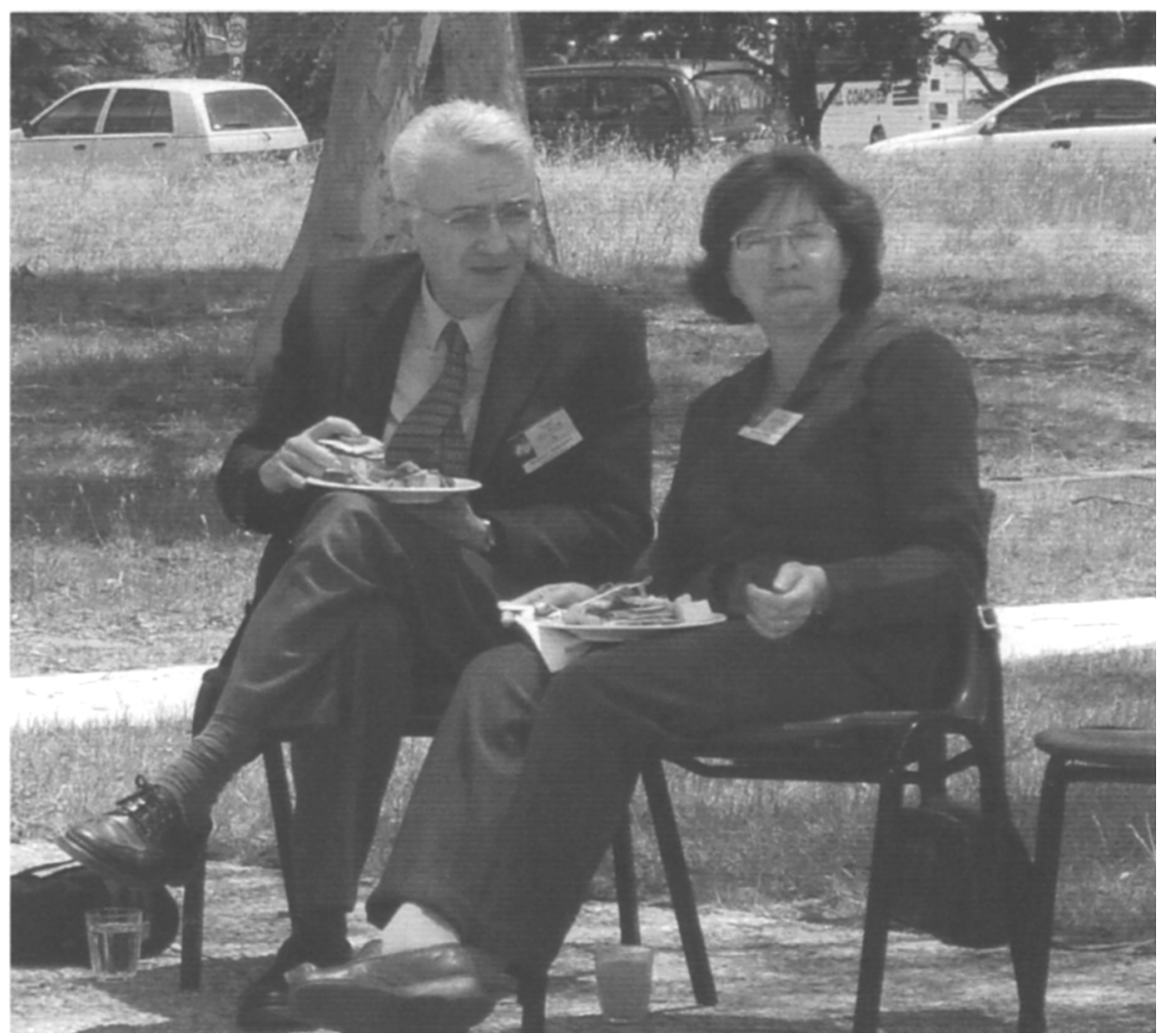

Manuel Peimbert and Miriam Peña relaxing at lunch outside the Academy dome (photo courtesy of O. de Marco). 